\title{
Shape and Contact Force Estimation of Continuum Manipulators using Pseudo Rigid Body Models
}

\author{
Venkatasubramanian Kalpathy Venkiteswaran ${ }^{\mathrm{a}}$, Jakub Sikorski ${ }^{\mathrm{a}}$, \\ Sarthak Misra ${ }^{\mathrm{a}, \mathrm{b}}$ \\ ${ }^{a}$ Surgical Robotics Laboratory, Department of Biomechanical Engineering, University of \\ Twente, 7500 AE Enschede, The Netherlands \\ ${ }^{b}$ Department of Biomedical Engineering, University of Groningen and University Medical \\ Centre Groningen, 9713 GZ Groningen, The Netherlands
}

\begin{abstract}
Accurate estimation of deformed shape and reaction forces poses a significant challenge in modeling and control of continuum manipulators interacting with their environment. This paper presents a framework for the analysis of a continuum manipulator subjected to multiple external loads using pseudo rigid body (PRB) modelling method. A PRB model with six degrees of freedom is developed, and the statics equations for a manipulator with multiple loads are presented, followed by the approach for determining the optimal model parameters. Dimensionless constants are used to enable scaling the model to manipulators of different lengths and materials. The approach is first validated by a comparison with Finite Element Analysis, and the results show a low error in estimating the tip deflection (less than $1 \%$ of the manipulator length). A magnetically-actuated manipulator driven by an array of electromagnetic coils is used for experimental validation. The optimization for a single segment of the manipulator is performed with experimental data, with an average error of 2.2 $\mathrm{mm}$ in calculating the tip position. An experimental setup for a manipulator with multiple loads is created by introducing displacement constraints with fixtures connected to multi-axis force sensors. The model shows good agreement with quasi-static experiments in estimating the reaction forces and predicting the deformed shape of the manipulator. The average error in force estimation is $61 \mathrm{mN}$, while the average error in estimating tip position is $2.8 \mathrm{~mm}$ for a manipulator length of $82 \mathrm{~mm}$.
\end{abstract}

Keywords: Continuum manipulator, pseudo-rigid-body model, force estimation, magnetic actuation, surgical robotics

Email addresses: v.kalpathyvenkiteswaran@utwente.nl

(Venkatasubramanian Kalpathy Venkiteswaran), j.sikorski@utwente.nl (Jakub Sikorski), s.misra@utwente.nl (Sarthak Misra) 


\section{INTRODUCTION}

Continuum manipulators provide significant advantages over traditional rigidlink robots in terms of locomotion and dexterity. They are particularly useful in navigating constrained and cluttered environments due to their inherent large number of degrees of freedom [1]. These characteristics make them conducive to exploratory operations such as search and rescue. Soft and compliant manipulators utilize the deformation of elastic elements to achieve motion [2]. With compliance matching, they can be safely used in intra-operative procedures since there is little risk of tissue damage, which makes them suitable for minimally invasive surgery (MIS) [3, 4].

However, the structural redundancy (multiple degrees of freedom) of continuum manipulators renders them difficult to model and control. Accurate modeling of such systems can be challenging due to the nonlinearities arising from material properties and large deformation. Constant-curvature approximations help reduce the complexity associated with continuum motion, but are limited to specific applications $[5,6]$. Another approach is to utilize Cosserat rods to develop geometrically-exact models $[7,8]$. Previously, Tang et al. used this method for estimating the shape of catheters and guidewires including surface friction, with experimental validation for small deflections [9]. Till et al. analyzed a wire-driven parallel manipulator [10]. Force estimation in continuum devices has been traditionally limited to single loads at the tip, whereas in reality contact may occur anywhere along the manipulator length [11],[12]. Recent work by Coevoet et al. and Zhang et al. has studied shape and force estimation under contact for soft manipulators using a Finite Element Method (FEM) model [13],[14].

In this paper, we present a pseudo rigid body modelling approach for estimation of deflection and shape of a magnetically-actuated continuum manipulator, including environmental loads (Fig. 1). This is demonstrated through experimental validation of deflection and shape calculation in 3D for quasi-static scenarios, including estimation of contact and non-contact loads that affect the motion of the manipulator. The framework used in this work can be adapted for other soft or continuum manipulators.

Pseudo rigid body (PRB) models are numerical approximations of flexible elements with springs for capturing the compliance and rigid links for estimating the kinematics of the system [15]. PRB models can be extended to encompass a variety of mechanical deformation characteristics, and allow for simplified definitions of compliant elements to speed up computation [16]. This also enables the models to be used for real-time control through inverse kinematics equations based on rigid-body dynamics. Khoshnam et al. previously used a PRB model for analysis of planar deflection of catheters [17]. Greigarn et al. also used a PRB model for catheter analysis, but it was limited to a specific catheter design and did not include interaction with the environment or the effect of gravity $[18,19]$. Previous work by Roesthuis and Misra used a constant-curvature approach combined with rigid-link modeling, but was restricted to manipulators undergoing planar deformations, and subjected only to moment loads [20]. 


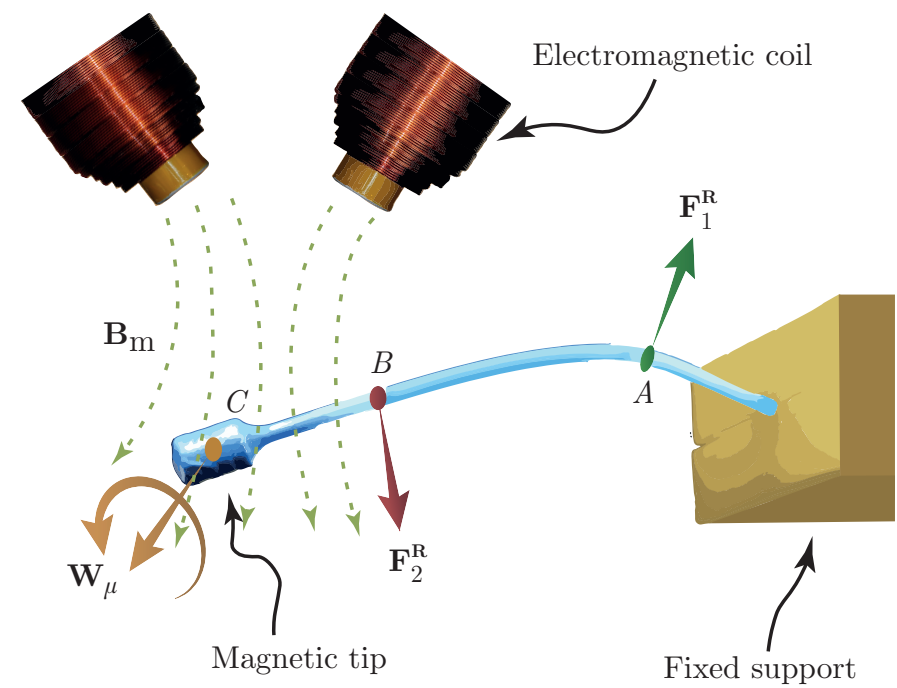

Figure 1: A continuum manipulator subjected to multiple loads is shown. The actuation wrench $\left(\mathbf{W}_{\mu} \in \mathbb{R}^{6}\right)$ is generated due to the interaction between the external magnetic field $\left(\mathbf{B}_{\mathbf{m}} \in \mathbb{R}^{3}\right)$ and the dipole at the magnetic tip $(C)$. Displacement boundary conditions are enforced at points $(A$ and $B)$ along the length of the manipulator, resulting in reaction forces $\left(\mathbf{F}_{1}^{\mathbf{R}} \in \mathbb{R}^{3}\right.$ and $\left.\mathbf{F}_{2}^{\mathbf{R}} \in \mathbb{R}^{3}\right)$. A framework for the analysis of continuum manipulators subjected to multiple loads using pseudo rigid body models is presented in this paper.

Huang et al. used a planar PRB model for analysis of a cable-driven continuum manipulator [21]. Open and closed-loop experiments were performed on a magnetic catheter by Edelmann et al., but required prior knowledge of external loads for numerical integration using Cosserat rods [22].

The PRB model presented here is generally applicable (in 3D) to manipulators made of flexible materials with a high aspect ratio (length to cross-section diameter). The framework also incorporates the response of the system to multiple external loads. This is demonstrated through the estimation of manipulator shape and contact forces with the environment, through both Finite Element Analysis (FEA) and experiments. The discussion is limited to static conditions, but the model is applicable to dynamic environments, and can handle large deformations. The model is defined with dimensionless parameters to enable it to be extended to other loading scenarios. The PRB model defines the equations of motion in a simple format that can be solved in real-time for closed-loop control. Compared to other methods in literature, the model can be fitted to a wide range of deformation characteristics even when accurate information about system properties is a priori unavailable.

The experiments for validating the methodology are conducted with a magnetic catheter made from a thermoplastic elastomer, for minimally invasive surgery. Magnetic actuation has the advantage of being non-contact and nonhazardous, which makes it suitable for surgical applications [23]. It also has the benefit of being frictionless, compared to conventional cable-driven systems 
[24]. Magnetic catheters have been demonstrated to improve surgical efficiency through better control during catheter guidance [25]. In this work, the actuation is achieved using an array of six electromagnetic coils called BigMag [26]. The coils can rotate around a spherical workspace with a diameter of approximately $10 \mathrm{~cm}$. The system is capable of producing magnetic fields of specified magnitude and direction at any point within the workspace. Two cameras are also present in the setup which facilitate the sensing of the manipulator position and shape using stereo vision techniques.

The rest of the paper is laid out as follows. Section 2 details the derivation of the PRB model using screw theory, followed by the procedure for determining the optimal values of the PRB parameters in Sec. 3. The results of the optimization are tabulated, with explanations and preliminary validation. Section 4 describes the experimental setup to test the accuracy of the model and the results from the experiments. This is followed by a discussion of the contributions of the work, and ideas for improving the accuracy. The last section summarizes the key points of the work presented here, and also looks at some potential avenues for future work.

\section{PSEUDO-RIGID-BODY MODEL}

In this section, a pseudo rigid body model is presented for determining the configuration of a flexible manipulator in three-dimensional space, under the influence of external loads. The manipulator analyzed here is assumed to undergo quasi-static motion, and has a large ratio of length to diameter with predominantly bending characteristics. The framework for deriving the model parameters and the method of analysis is generally applicable to manipulators with similar deformation behavior.

Figure 2 shows a flexible manipulator in static equilibrium with loads $\left(\mathbf{W}_{k} \in\right.$ $\left.\mathbb{R}^{6}(k=1,2,3)\right)$ at points $\left(\mathbf{P}_{k} \in \mathbb{R}^{3}\right)$ along its length, fixed at end $\mathbf{P}_{0}$. Each segment $\left(S_{k}\right)$ taken in isolation is in equilibrium with a load $\left(\mathbf{W} \in \mathbb{R}^{6}\right)$ at its ends. In this paper, a PRB model with six degrees of freedom is used to approximate a given segment. The PRB model used here has four rigid links of a given length $\left(l_{i} \in \mathbb{R}^{+}\right)$, connected by three joints, each with two degrees of freedom. Each joint $(i)$ at point $\left(\mathbf{r}_{i} \in \mathbb{R}^{3}\right)$ undergoes two angular deformations, first by angle $\left(\eta_{i} \in \mathbb{S}\right)$ about the $y$-axis, followed by $\left(\theta_{i} \in \mathbb{S}\right)$ about the $z$ axis. The rotational stiffness of each degree of freedom is defined by constants $\left(K_{\eta_{i}}, K_{\theta_{i}} \in \mathbb{R}^{+}\right)$, corresponding to the respective joint angles $\left(\eta_{i}, \theta_{i}\right)$.

The transformation from joint $(i)$ to joint $(i+1), \mathbf{T}_{i}^{i+1} \in \mathrm{SE}(3)$ is given by

$$
\mathbf{T}_{i}^{i+1}=\left[\begin{array}{cccc}
\mathrm{c}_{\eta_{i}} \mathrm{c}_{\theta_{i}} & -\mathrm{c}_{\eta_{i}} \mathrm{~s}_{\theta_{i}} & \mathrm{~s}_{\eta_{i}} & l_{i} \\
\mathrm{~s}_{\eta_{i}} & \mathrm{c}_{\theta_{i}} & 0 & 0 \\
-\mathrm{s}_{\eta_{i}} \mathrm{c}_{\theta_{i}} & \mathrm{~s}_{\eta_{i}} \mathrm{~s}_{\theta_{i}} & \mathrm{c}_{\eta_{i}} & 0 \\
0 & 0 & 0 & 1
\end{array}\right]
$$

where $\mathrm{s}_{*}$ and $\mathrm{c}_{*}$ are the sine and cosine of angle $(*)$, respectively. The coordi- 


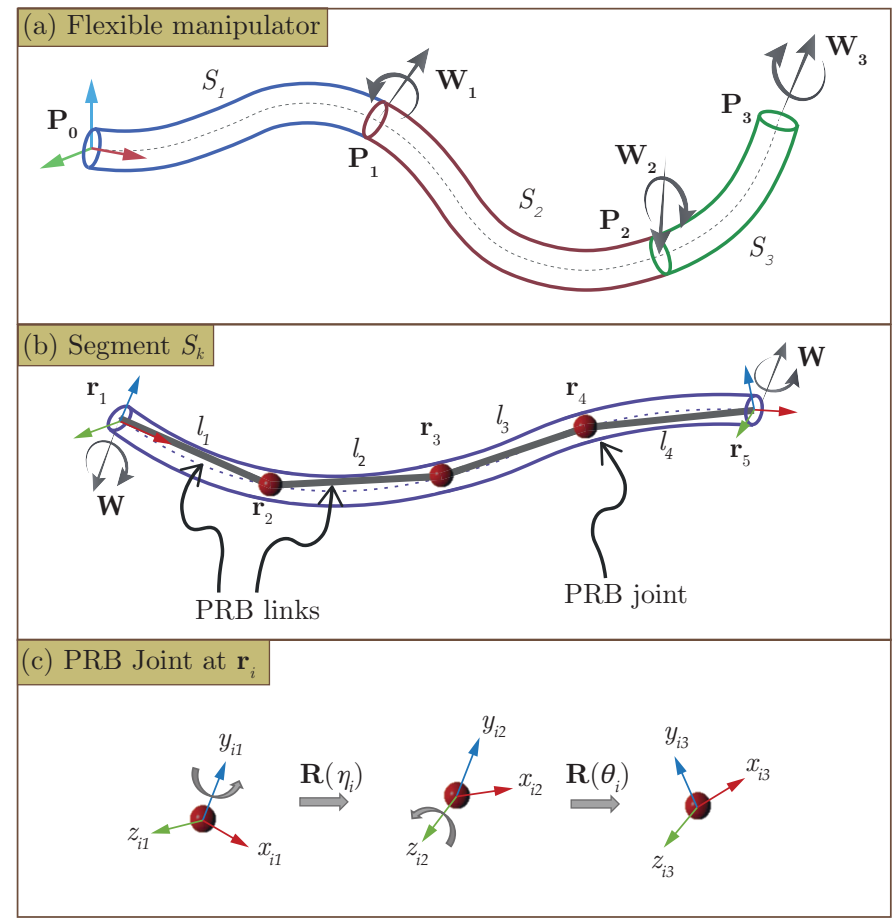

Figure 2: A schematic of a 3D pseudo rigid body model for analysis of a flexible manipulator is shown. (a) The manipulator is fixed at one end $\left(\mathbf{P}_{0} \in \mathbb{R}^{3}\right)$, and deforms upon being subjected to loads $\left(\mathbf{W}_{k} \in \mathbb{R}^{6}\right)$ at points $\left(\mathbf{P}_{k} \in \mathbb{R}^{3}\right)(k=1,2,3)$ along its length. The manipulator is partitioned into segments $\left(S_{1}, S_{2}\right.$ and $\left.S_{3}\right)$ between two successive loads. (b) Each segment $\left(S_{k}\right)$ is in static equilibrium with tip load $\left(\mathbf{W} \in \mathbb{R}^{6}\right)$. Using the pseudo rigid body modeling approach, the segment can be replaced by a series of rigid links (with length of link $(i)$ defined as $l_{i} \in \mathbb{R}^{+}$) connected by joints. In this work, a four-link pseudo rigid body model is used, with joints at $\mathbf{r}_{i} \in \mathbb{R}^{3} \quad(i=1,2,3,4,5)$. (c) There are two successive intrinsic rotations at each joint: first by an angle $\left(\eta_{i} \in \mathbb{S}\right)$ about the $y$-axis, followed by an angle $\left(\theta_{i} \in \mathbb{S}\right)$ about the $z$-axis (represented as $\mathbf{R}\left(\eta_{i}\right) \in \mathrm{SO} 3$ and $\mathbf{R}\left(\theta_{i}\right) \in \mathrm{SO} 3$, respectively).

nates of each joint $\left(\mathbf{r}_{i}\right)$ are calculated as

$$
\left[\begin{array}{c}
\mathbf{r}_{i} \\
1
\end{array}\right]=\prod_{j=1}^{i-1} \mathbf{T}_{j}^{j+1} \cdot\left[\begin{array}{c}
\mathbf{r}_{1} \\
1
\end{array}\right] .
$$

In the above equation, the angles related to the first joint $\left(\eta_{1}\right.$ and $\left.\theta_{1}\right)$ have zero magnitude since the first link is assumed to be rigidly fixed to the base frame.

The geometric twist between two successive degrees of freedom is calculated from the rotation axis $\left(\omega_{*} \in \mathbb{R}^{3}\right)$ and the position vector $\left(q_{*} \in \mathbb{R}^{3}\right)$. For joint $(i)$,

$$
\begin{gathered}
\mathbf{q}_{\eta_{i}}=\left(\begin{array}{lll}
l_{i-1} & 0 & 0
\end{array}\right)^{T} \\
\mathbf{q}_{\theta_{i}}=\left(\begin{array}{lll}
0 & 0 & 0
\end{array}\right)^{T}
\end{gathered}
$$




$$
\begin{aligned}
& \boldsymbol{\omega}_{\eta_{i}}=\left(\begin{array}{lll}
0 & 1 & 0
\end{array}\right)^{T}, \\
& \boldsymbol{\omega}_{\theta_{i}}=\left(\begin{array}{lll}
0 & 0 & 1
\end{array}\right)^{T},
\end{aligned}
$$

which allows us to define the associated twists $\left(\boldsymbol{\zeta}_{\eta_{i}}, \boldsymbol{\zeta}_{\theta_{i}} \in \mathbb{R}^{6}\right)$ as

$$
\begin{aligned}
\boldsymbol{\zeta}_{\eta_{i}} & =\left[\begin{array}{c}
\mathbf{q}_{\eta_{i}} \times \boldsymbol{\omega}_{\eta_{i}} \\
\boldsymbol{\omega}_{\eta_{i}}
\end{array}\right]=\left[\begin{array}{llllll}
0 & 0 & l_{i-1} & 0 & 1 & 0
\end{array}\right]^{T}, \\
\boldsymbol{\zeta}_{\theta_{i}} & =\left[\begin{array}{c}
\mathbf{q}_{\theta_{i}} \times \boldsymbol{\omega}_{\theta_{i}} \\
\boldsymbol{\omega}_{\theta_{i}}
\end{array}\right]=\left[\begin{array}{llllll}
0 & 0 & 0 & 0 & 0 & 1
\end{array}\right]^{T} .
\end{aligned}
$$

The spatial manipulator Jacobian is used to derive the statics equations for the PRB model [27]. The set of all the angles $\left(\Theta \in \mathbb{S}^{6}\right)$ that define the configuration of the PRB model is

$$
\Theta=\left[\eta_{2}, \theta_{2}, \eta_{3}, \theta_{3}, \eta_{4}, \theta_{4}\right]^{T} .
$$

The Jacobian $\left(\mathbf{J}_{\Theta} \in \mathbb{R}^{6 \times 6}\right)$ is given by

$$
\mathbf{J}_{\Theta}=\left[\begin{array}{llllll}
\boldsymbol{\zeta}_{\eta_{2}}^{\prime} & \boldsymbol{\zeta}_{\theta_{2}}^{\prime} & \boldsymbol{\zeta}_{\eta_{3}}^{\prime} & \boldsymbol{\zeta}_{\theta_{3}}^{\prime} & \boldsymbol{\zeta}_{\eta_{4}}^{\prime} & \boldsymbol{\zeta}_{\theta_{4}}^{\prime}
\end{array}\right]
$$

where

$$
\begin{aligned}
\boldsymbol{\zeta}_{\eta_{i}}^{\prime} & =\operatorname{Ad}\left(\left.\mathbf{T}_{1}^{2} \ldots \mathbf{T}_{i-2}^{i-1}\right|_{l_{i-1}=0}\right) \boldsymbol{\zeta}_{\eta_{i}} \\
\boldsymbol{\zeta}_{\theta_{i}}^{\prime} & =\operatorname{Ad}\left(\left.\mathbf{T}_{1}^{2} \ldots \mathbf{T}_{i-1}^{i}\right|_{\substack{\eta_{i}=0 \\
l_{i}=0}}\right) \boldsymbol{\zeta}_{\theta_{i}},
\end{aligned}
$$

where $\operatorname{Ad}(\mathbf{T})$ represents the adjoint transformation associated with the transformation matrix $\mathbf{T}$.

The set of internal torques in the PRB model $\left(\boldsymbol{\tau} \in \mathbb{R}^{6}\right)$ is represented as

$$
\boldsymbol{\tau}=\left[\begin{array}{ll}
K_{\eta_{i}} \eta_{i} & K_{\theta_{i}} \theta_{i}
\end{array}\right] \quad i=2,3,4 .
$$

The load wrench $(\mathbf{W})$ can be decomposed into a force $\left(\mathbf{F} \in \mathbb{R}^{3}\right)$ and a moment $\left(\mathbf{M} \in \mathbb{R}^{3}\right)$, and defined in the spatial reference frame as

$$
\mathbf{W}=\left[\begin{array}{c}
\mathbf{F} \\
\mathbf{M}
\end{array}\right]+\left[\begin{array}{c}
0_{3 \times 1} \\
\mathbf{r}_{5} \times \mathbf{F}
\end{array}\right]
$$

The above definition of the external loads allows the inclusion of any form of actuation or contact that can be represented as a point load. The static behavior of the PRB model can be determined using the following equation.

$$
\boldsymbol{\tau}=\left(\mathbf{J}_{\Theta}\right)^{T} \mathbf{W} .
$$

The above equation is a system of six nonlinear algebraic equations that can be solved to obtain the values of the six variables $\left(\eta_{i}, \theta_{i}: i=2,3,4\right)$ given any load wrench acting at the tip. 
Equation (14) can be extended to a system having multiple loads, such as the one shown in Fig. 2(a) with three loads acting on it. Each segment $\left(S_{k}\right)$ can be replaced by the 6-DOF PRB model for analysis, with the ends of adjacent segments rigidly attached to one another. The statics equations for the system are given by

$$
\left[\begin{array}{c}
\boldsymbol{\tau}_{1} \\
\boldsymbol{\tau}_{2} \\
\boldsymbol{\tau}_{3}
\end{array}\right]=\left[\begin{array}{ccc}
\left(\mathbf{J}_{\boldsymbol{\Theta}_{1}}\right)^{T} & \left(\mathbf{J}_{\boldsymbol{\Theta}_{1}}\right)^{T} & \left(\mathbf{J}_{\boldsymbol{\Theta}_{1}}\right)^{T} \\
0 & \left(\mathbf{J}_{\boldsymbol{\Theta}_{2}}\right)^{T} & \left(\mathbf{J}_{\boldsymbol{\Theta}_{2}}\right)^{T} \\
0 & 0 & \left(\mathbf{J}_{\boldsymbol{\Theta}_{3}}\right)^{T}
\end{array}\right]\left[\begin{array}{c}
\mathbf{W}_{\mathbf{1}} \\
\mathbf{W}_{\mathbf{2}} \\
\mathbf{W}_{\mathbf{3}}
\end{array}\right],
$$

where $\mathbf{J}_{\boldsymbol{\Theta}_{k}}$ is the Jacobian matrix associated with segment $S_{k}$, and $\boldsymbol{\tau}_{k}$ is the corresponding set of internal torques.

\section{PARAMETER OPTIMIZATION}

In this section, the values of the constant PRB parameters $\left(l_{i}, K_{\eta_{i}}\right.$ and $\left.K_{\theta_{i}}\right)$ are determined using an optimization process. This is accomplished using data obtained from other controlled and verifiable methods, such as high-fidelity simulations (theoretical data) and experiments (empirical data). Once the parameter values for a single PRB segment are calculated using this approach, manipulators of similar materials and geometries can be modeled using the same parameters. The optimal parameter values for the single segment model are used in Sec. 4 to analyze a manipulator with multiple loads exerted on it .

\subsection{Optimization Framework}

The PRB parameters are defined with dimensionless variables as given below.

$$
\begin{aligned}
l_{i} & =\gamma_{i} L, \\
K_{\eta_{j}} & =k_{\eta_{j}} \frac{E I}{L}, \\
K_{\theta_{j}} & =k_{\theta_{j}} \frac{E I}{L},
\end{aligned}
$$

where $i=1,2,3,4, j=2,3,4, E I$ represents the flexural rigidity (product of elastic modulus and area moment of inertia) and $L$ is the length of the manipulator segment. This allows the model to be extended to similar manipulators by using appropriate material properties and geometric dimensions.

The optimization process calculates the best values of the PRB parameters so that the model closely matches the deformation behavior of the manipulator segment. From the verifiable data, a set of $N$ static loading cases is extracted to be used as the basis for optimization[28]. The error of the PRB model in modeling a specific loading case $(k)$ is defined as

$$
e_{k}=\left\|\mathbf{Y}_{k}-\mathbf{Y}_{k}^{P R B}\right\|,
$$


where $\mathbf{Y}_{k}^{P R B}$ is the coordinates of the tip $\left(\mathbf{r}_{\mathbf{5}}\right)$ calculated using the PRB model, and the corresponding results from the verifiable data are represented by $\mathbf{Y}_{k}$. If $\mathbf{X}$ is the set of PRB parameters to be optimized, the optimization problem can be defined as

$$
\begin{aligned}
& \text { Minimize } \\
& \text { where } \\
& \text { subject to } \\
& \mathbf{X}=\left\{\gamma_{i}, k_{\eta_{j}}, k_{\theta_{j}}\right\}, \\
& i=1,2,3,4 \quad j=2,3,4, e_{k}, \mathbf{X}_{l b} \leq \mathbf{X} \leq \mathbf{X}_{u b}, \\
& \sum_{i=1}^{4} \gamma_{i}=1,
\end{aligned}
$$

where $\mathbf{X}_{l b}$ and $\mathbf{X}_{u b}$ are the lower and upper bounds of the variables under optimization, respectively.

In this work, the model is defined to be symmetric about the middle joint $\left(\mathbf{r}_{3}\right)$, which has two advantages. Firstly, it reduces the number of variables in the optimization, and secondly, it allows the implementation of boundary conditions at either end without affecting the analysis. This leads to the following relations.

$$
\begin{gathered}
\gamma_{1}=\gamma_{4} \quad(21) \quad k_{\eta_{2}}=k_{\eta_{4}} \quad(22) \quad k_{\theta_{2}}=k_{\theta_{4}} \\
\gamma_{2}=\gamma_{3}=0.5-\gamma_{1} .
\end{gathered}
$$

Therefore, there are five independent PRB parameters to be determined, namely $\gamma_{1}, k_{\eta_{2}}, k_{\eta_{3}}, k_{\theta_{2}}$ and $k_{\theta_{3}}$. The optimization was performed using the function fmincon in MATLAB (Version 9.2.0.556344, Mathworks Inc., Natick, Massachusetts, U.S.A.).

\subsection{Validation with Finite Element Analysis}

The proposed method using the PRB model is first validated using FEA. A continuum manipulator is modeled in Ansys Workbench (Version 16.2, Ansys Inc., Canonsburg, Pennsylvania, U.S.A.) using BEAM188 elements (2-node beam element in 3D) of $1 \mathrm{~mm}$ length. The optimization of the PRB parameters is initially performed for a single segment. Force and moment loads are applied at the tip of the manipulator, while the other end is fixed. The maximum and minimum values of the forces $\left(F_{x}, F_{y}, F_{z}\right)$ are $-4 \mathrm{mN}$ and $4 \mathrm{mN}$, respectively, while those for the moments $\left(M_{y}, M_{z}\right)$ are $-250 \mathrm{mN} \mathrm{mm}$ and $250 \mathrm{mN} \mathrm{mm}$, respectively. Torsional moments $\left(M_{x}\right)$ are not applied. A total of $N=7776$ data points are obtained for a manipulator of length $50 \mathrm{~mm}$, with an elastic modulus $(E)$ of $350 \mathrm{MPa}$ and a bending moment of inertia $(I)$ of $4.91 \times 10^{-} 2 \mathrm{~mm}^{4}$.

The framework described in Sec. 3.1 is used to determine the optimal values of the PRB parameters, which are given in Table ??. The average error in 
estimation of the tip position by the model compared to the FEA data is 0.488 $\mathrm{mm}$ (less than $1 \%$ of the manipulator length). Four deflected positions of the manipulator and the corresponding PRB model are shown in Fig. 3.

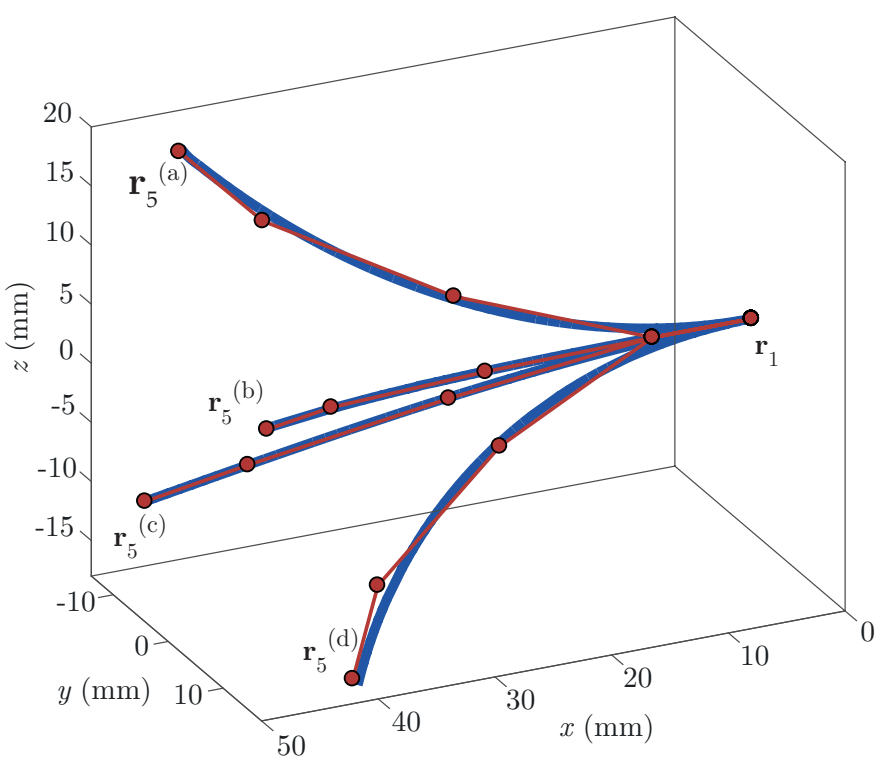

Figure 3: Comparison of optimized pseudo rigid body model (red) to manipulator shape from Finite Element Analysis (blue) calculated using Ansys Workbench (Version 16.2, Ansys Inc., Canonsburg, Pennsylvania, U.S.A.) for a single segment with tip loads. Four example configurations are shown $(\mathrm{a}, \mathrm{b}, \mathrm{c}, \mathrm{d})$. The manipulator is fixed at $\mathbf{r}_{0}$ and loads are applied at the tip locations $\left(\mathbf{r}_{5}^{*}\right)$. The length of the undeformed manipulator is $50 \mathrm{~mm}$. The tip loads in the different configurations are: (a) $\mathbf{F}=(4,-2.5,2.5) \mathrm{mN}, \mathbf{M}=(0,-250,-150) \mathrm{mN} \mathrm{mm}$, (b) $\mathbf{F}=(-4,-4,-4) \mathrm{mN}, \mathbf{M}=(0,0,0) \mathrm{mN} \mathrm{mm},(\mathrm{c}) \mathbf{F}=(0,1,2) \mathrm{mN}, \mathbf{M}=(0,0,150) \mathrm{mN} \mathrm{mm}$, (d) $\mathbf{F}=(-3,1,0) \mathrm{mN}, \mathbf{M}=(0,200,-100) \mathrm{mN} \mathrm{mm}$.

The same parameter values are then used to analyze a manipulator with multiple segments. Point loads are applied at specific positions along a length of $110 \mathrm{~mm}$. The multi-segment model described in Eqn. (15) is used to analyze the system, and the results from the PRB model and FEA are plotted in Fig. 4. The plot shows good agreement between the two sets of results, and the shape of the manipulator is estimated by the PRB model by an mean error of $0.36 \mathrm{~mm}$ along its length across the three loading cases. These results demonstrate that once the optimal PRB parameters for a single segment are determined, they can be used for similar manipulators of different lengths subjected to multiple loads.

\section{EXPERIMENTS AND RESULTS}

In this section, a magnetically-actuated surgical catheter is used to illustrate the application of the PRB model through experiments. At first, the PRB 
Table 1: Optimal values of dimensionless pseudo rigid body model parameters for minimizing the model error based on data from Finite Element Analysis. The parameters relate to the length of the pseudo rigid body segment $\left(l_{1}=\gamma_{1} L\right.$, where $L$ is the length of the manipulator segment) and stiffness of the joints $\left(K_{\eta_{j}}=k_{\eta_{j}} \frac{E I}{L}, K_{\theta_{j}}=k_{\theta_{j}} \frac{E I}{L}, j=2,3\right.$, where $E$ is the elastic modulus and $I$ is the second moment of area of the cross section of the manipulator). The optimization is performed over $N=7776$ data points as described in Sec. 3.2. The average error is estimating the tip position is $0.98 \%$ of the manipulator length.

\begin{tabular}{ccccc}
\hline$\gamma_{1}$ & $k_{\eta_{2}}$ & $k_{\eta_{3}}$ & $k_{\theta_{2}}$ & $k_{\theta_{3}}$ \\
\hline 0.1699 & 2.5064 & 4.8339 & 2.4914 & 5.0303 \\
\hline
\end{tabular}

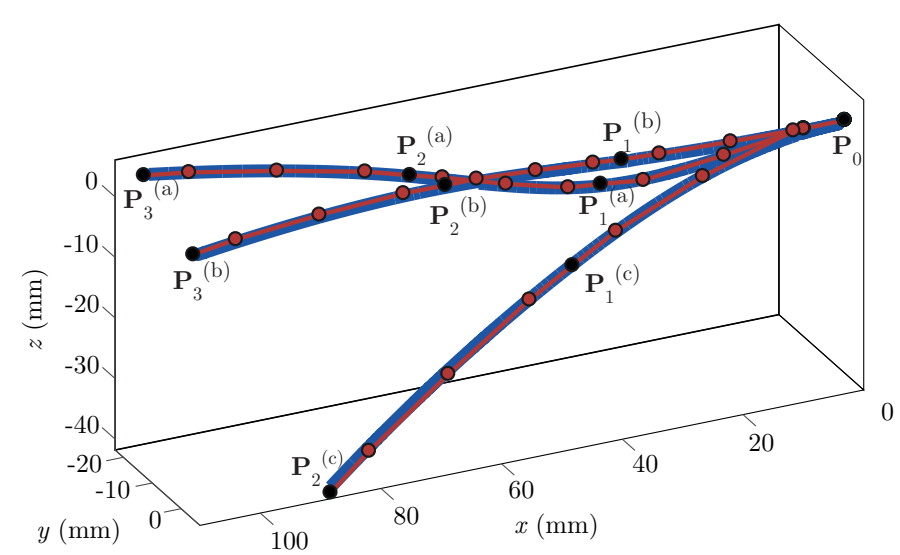

Figure 4: Comparison of optimized pseudo rigid body model (red) to manipulator shape from Finite Element Analysis (blue) for multiple external loads. Three example configurations are shown $(\mathrm{a}, \mathrm{b}, \mathrm{c})$. The manipulator is fixed at $\mathbf{P}_{0}$ and the locations of the loads are marked $\mathbf{P}_{1}^{*}, \mathbf{P}_{2}^{*}$ and $\mathbf{P}_{3}^{*}$. The length of the undeformed manipulator is $110 \mathrm{~mm}$. The loads applied in the various configurations are: (a) : $\mathbf{F}_{1}=(0,5.15,0) \mathrm{mN}, \mathbf{F}_{2}=(0,0,4.91) \mathrm{mN}, \mathbf{F}_{3}=$ $(0,0,-1) \mathrm{mN}, \mathbf{M}_{3}=(0,100,-100) \mathrm{mN} \mathrm{mm}, \quad(\mathrm{b}): \mathbf{F}_{1}=(0,2.69,-2.66) \mathrm{mN}, \mathbf{F}_{2}=$ $(0,0,-15.0) \mathrm{mN}, \mathbf{F}_{3}=(0,0,-1) \mathrm{mN}, \mathbf{M}_{3}=(0,100,-100) \mathrm{mN} \mathrm{mm}, \quad$ (c) $\quad: \quad \mathbf{F}_{1}=$ $(0,5.15,0) \mathrm{mN}, \mathbf{F}_{2}=(0,0,-1) \mathrm{mN}, \mathbf{M}_{2}=(0,100,-100) \mathrm{mN} \mathrm{mm}$. . Note that configuration (c) has only two points of loading. 
parameters are optimized for a segment with point loads at its free end, and then the same values are used to predict the deformation of a similar device in a multiple-load scenario.

\subsection{Experiment setup}

The experiments are performed using an array of electromagnetic coils (BigMag [26]). The manipulator is a hollow tube (diameter $\phi_{\text {outer }}=2 \mathrm{~mm}, \phi_{\text {inner }}=$ $1.2 \mathrm{~mm}$ ) made of an elastomer (PEBAX 6663). Four Neodymium ring magnets (height $h=2 \mathrm{~mm}$, diameter $\phi_{\text {outer }}=6 \mathrm{~mm}, \phi_{\text {inner }}=2 \mathrm{~mm}$, and mass $m=0.38$ g) are attached to the tip of the tube. The magnetic dipole $\left(\boldsymbol{\mu} \in \mathbb{R}^{3},|\boldsymbol{\mu}|=0.176\right.$ $\mathrm{A} / \mathrm{m}^{2}$ ) acts along the axis of the ring magnets. The electromagnet coil array is capable of producing a specified magnetic field $\left(\mathbf{B}_{\mathbf{m}} \in \mathbb{R}^{3}\right)$ at the tip of the manipulator. The field produces a force $\left(\mathbf{F}_{\mu} \in \mathbb{R}^{3}\right)$ and a moment $\left(\mathbf{M}_{\mu} \in \mathbb{R}^{3}\right)$ given by

$$
\begin{array}{r}
\mathbf{F}_{\mu}=\nabla\left(\boldsymbol{\mu} \cdot \mathbf{B}_{\mathbf{m}}\right) \\
\mathbf{M}_{\mu}=\boldsymbol{\mu} \times \mathbf{B}_{\mathbf{m}},
\end{array}
$$

where $\boldsymbol{\mu}$ is aligned with the axis of the manipulator tip. The mass of the magnets also produces a gravity force $\left(\mathbf{F}_{g}=m \mathbf{g} \in \mathbb{R}^{3}\right)$ at the same point $\left(\mathrm{g} \in \mathbb{R}^{3}\right.$ is the acceleration due to gravity). The mass of the manipulator is neglected since it is significantly smaller than that of the magnets.

The total force and moment at the manipulator tip (as defined in Eqn. (13)) are given by

$$
\begin{array}{r}
\mathbf{F}=\mathbf{F}_{\mu}+\mathbf{F}_{g}, \\
\mathbf{M}=\mathbf{M}_{\mu},
\end{array}
$$

and is used to actuate the manipulator. The experimental setup is shown in Fig. 5. The manipulator shape is tracked with a stereo vision system and reconstructed using a technique similar to the one described by Camarillo et al. [29].

\subsection{Optimization Results}

The magnetic setup described above is used to generate a set of data for determining the PRB parameters for a single manipulator segment of length $52 \mathrm{~mm}$. The magnetic field is applied to obtain deflected manipulator configurations using vision-based tracking from the cameras, and data (magnetic field and manipualtor shape for $N=360$ points) is collected. The range of loads used in the experiment is given in Table 2. The optimization framework is applied to fit the model and the resulting PRB parameter values are given in Table 3. The optimized PRB model estimates the position of the manipulator tip with an error of $2.2 \pm 1.76 \mathrm{~mm}$ over the entire range of data. The high standard deviation arises from error at a few data points where the magnet is close to the electromagnetic coils (very large forces). The accuracy of the model is also illustrated through four representative manipulator configurations in Fig. 6. 


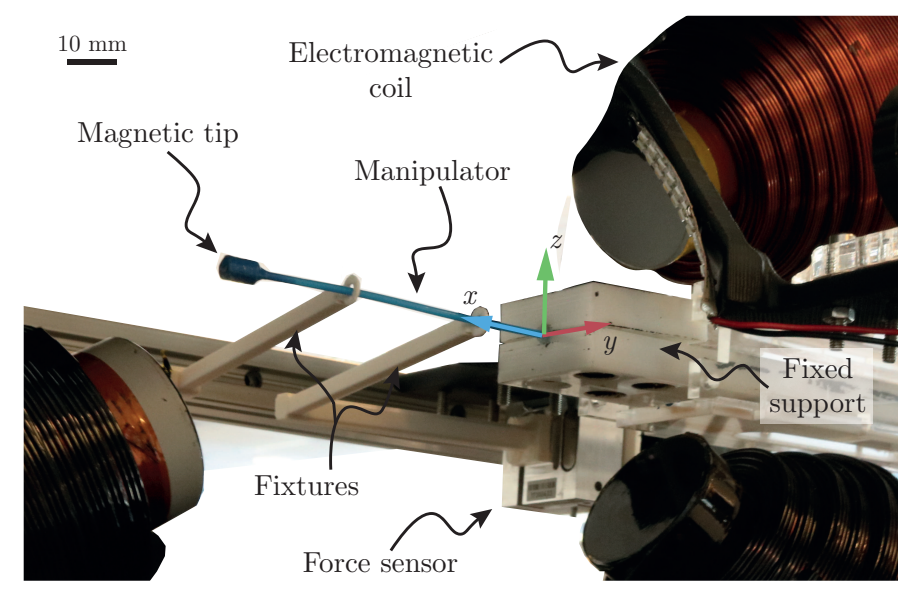

Figure 5: The experimental setup for validating the pseudo rigid body model, with a continuum manipulator actuated by an array of six electromagnetic coils (three are shown). The manipulator is fixed at one end (directions of the axes at the base frame are shown) and permanent magnets are attached to the other end. Fixtures are used to enforce displacement constraints for simulating a manipulator with environmental loads. The contact forces between the fixtures and the manipulator are measured using two three-axis force sensors (one is not in frame). The shape of the manipulator is captured in the $x y$ - and $x z$-planes using cameras.

Table 2: Minimum and maximum values of magnetic forces and moments applied to manipulator tip when collecting data for optimization of the pseudo rigid body model. The load values are calculated using Eqns. (25),(26) in the fixed spatial reference frame, with knowledge of the applied magnetic field and the direction of the magnetic dipole at the tip of the manipulator.

\begin{tabular}{lccc}
\hline & $F_{\mu}^{x}(\mathrm{mN})$ & $F_{\mu}^{y}(\mathrm{mN})$ & $F_{\mu}^{z}(\mathrm{mN})$ \\
\hline Minimum & -11.1 & -55.2 & -23.9 \\
Maximum & 9.46 & 27.9 & 30.4 \\
\hline & $M_{\mu}^{x}(\mathrm{mN} \mathrm{mm})$ & $M_{\mu}^{y}(\mathrm{mN} \mathrm{mm})$ & $M_{\mu}^{z}(\mathrm{mN} \mathrm{mm})$ \\
\hline Minimum & -270.4 & -1735.5 & -1687.4 \\
Maximum & 355.6 & 1630.1 & 1809.7 \\
\hline
\end{tabular}


Table 3: Optimal values of dimensionless pseudo rigid body model parameters for minimizing the model error based on experimental data. The parameters relate to the length of the pseudo rigid body segment $\left(l_{1}=\gamma_{1} L\right.$, where $L$ is the length of the manipulator segment) and stiffness of the joints $\left(K_{\eta_{j}}=k_{\eta_{j}} \frac{E I}{L}, K_{\theta_{j}}=k_{\theta_{j}} \frac{E I}{L}, j=2,3\right.$, where $E I$ represents the flexural rigidity of the manipulator). The optimization is performed over $N=360$ data points as described in Sec. 4.1.

\begin{tabular}{ccccc}
\hline$\gamma_{1}$ & $k_{\eta_{2}}$ & $k_{\eta_{3}}$ & $k_{\theta_{2}}$ & $k_{\theta_{3}}$ \\
\hline 0.1184 & 1.8895 & 5.6053 & 2.0106 & 5.0667 \\
\hline
\end{tabular}

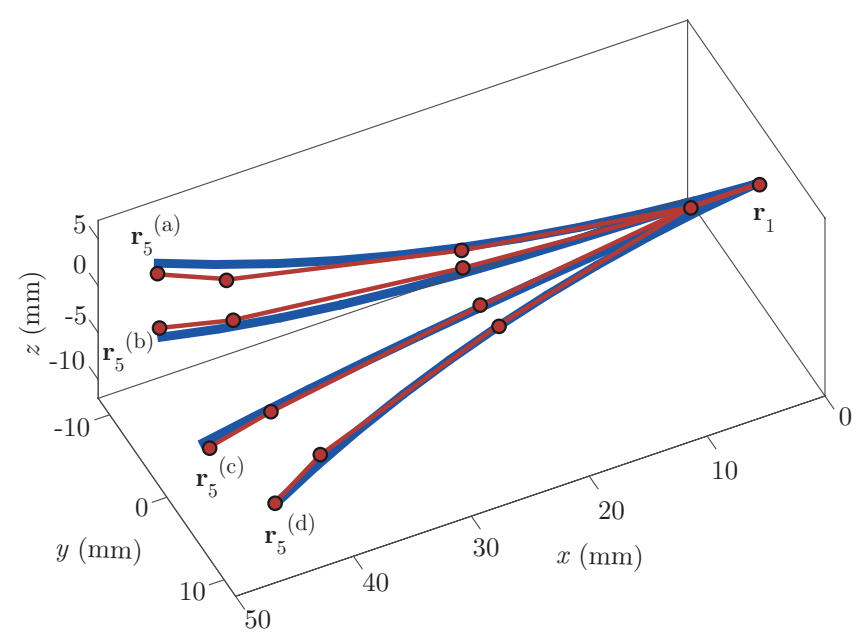

Figure 6: Comparison of optimized pseudo rigid body model (red) to manipulator shape from experiments (blue) for a single segment with tip loads. Four example configurations are shown ( $\mathrm{a}, \mathrm{b}, \mathrm{c}$ and $\mathrm{d}$ ). The manipulator is fixed at $\mathbf{r}_{1}$ and loads are applied at the tip locations $\left(\mathbf{r}_{5}^{*}\right.$, where $*$ is $(\mathrm{a}),(\mathrm{b}),(\mathrm{c})$ or $\left.(\mathrm{d})\right)$. The length of the undeformed manipulator is 52 $\mathrm{mm}$. The tip loads in the different configurations are: (a) $\mathbf{F}=(1.27,0.21,-1.62) \mathrm{mN}, \mathbf{M}=$ $(-1580,-780,-1360) \mathrm{mN} \mathrm{mm}$, (b) $\mathbf{F}=(1.27,0.83,-0.14) \mathrm{mN}, \mathbf{M}=(-160,0,-1330) \mathrm{mN}$ $\mathrm{mm}$, (c) $\mathbf{F}=(-0.10,1.43,-0.08) \mathrm{mN}, \mathbf{M}=(0,510,0) \mathrm{mN} \mathrm{mm},(\mathrm{d}) \mathbf{F}=(-4.32,-3.77,-6.07)$ $\mathrm{mN}, \mathbf{M}=(210,0,1500) \mathrm{mN} \mathrm{mm}$. 


\subsection{Multi-load experiments}

In this section, the application of the optimized PRB model for the analysis of a manipulator subjected to multiple loads is demonstrated. Similar to Fig. 2 (a), the manipulator is fixed at one end $\mathbf{P}_{0}$ and actuated by magnetic loads and gravity at end $\mathbf{P}_{3}$. Two displacement constraints are applied at points $\mathbf{P}_{1}$ and $\mathbf{P}_{2}$, leading to reaction forces $\mathbf{F}_{1}$ and $\mathbf{F}_{2}$. It is assumed that there are no moment reactions since there are no orientation constraints. Here, the analysis involves calculating the reaction forces and the manipulator shape, when the actuation loads and position of displacement constraints are known. If $\mathbf{F}_{3}, \mathbf{M}_{3}$, $\mathbf{P}_{1}$ and $\mathbf{P}_{2}$ are known, the PRB angles $\left(\boldsymbol{\Theta}_{i}, i=1,2,3\right)$, and the forces $\left(\mathbf{F}_{1}\right.$ and $\mathbf{F}_{2}$ ) can be determined using Eqns. (2) and (15), a system of 24 equations in as many unknowns. The equations were solved using f solve function in MATLAB (Version 9.2.0.556344).

The displacement constraints are applied using two fixtures, which are designed to constrain the manipulator only in the lateral direction ( $y$ and $z$ axes), while allowing slippage in the axial direction ( $x$ axis). Due to this, the $x$ component of the reaction forces is assumed to be zero for the analysis. The fixtures are printed using acrylonitrile butadiene styrene (ABS) and are attached to three-axis force sensors (Model number: K3D40 from ME-Meßsysteme GmbH, Hennigsdorf, Germany). The total length of the manipulator for this experiment is $82 \mathrm{~mm}$. The locations of the constraints $\left(\mathbf{P}_{1}\right.$ and $\left.\mathbf{P}_{2}\right)$ are calculated when the magnetic field is zero.

The experiment was run for 169 values of the actuation load, and the shape and force data were recorded. The forces were also estimated using the PRB model as described above (with the parameter values in Table 3 ), and the values obtained from the model were compared against those from the experiment in Fig. 7. The mean error in estimating the reaction forces was $140 \mathrm{mN}$ and 27 $\mathrm{mN}$ for the $y$ and $z$ components of $\mathbf{F}_{1}$, and $45 \mathrm{mN}$ and $32 \mathrm{mN}$ for $\mathbf{F}_{2}$.

The shape of the manipulator as defined by the PRB model was determined using 3D splines. The splines were then compared against the recorded shape data from the cameras at 25 points along the length of the manipulator. For a given frame $(i)$, the error in shape estimation $\left(e_{i}^{s} \in \mathbb{R}^{+}\right)$using the PRB model is calculated as

$$
e_{i}^{s}=\sum_{k=1}^{25}\left\|\mathbf{r}_{i, k}^{E}-\mathbf{r}_{i, k}^{P}\right\|
$$

where $\mathbf{r}_{i, k}^{E} \in \mathbb{R}^{3}$ and $\mathbf{r}_{i, k}^{P} \in \mathbb{R}^{3}$ refer to the coordinates of the points obtained from experiment and PRB model, respectively. The mean error in estimating the shape along the manipulator length was $1.2 \pm 0.23 \mathrm{~mm}$, also shown as a histogram in Fig. 8. The PRB model is plotted against the deformed manipulator configurations for two frames in Fig. 9.

\subsection{Discussion}

The error in estimation of the $y$-component of $\mathbf{F}_{1}$ is higher than others, which could be because the force is outside the range of loads used for the 

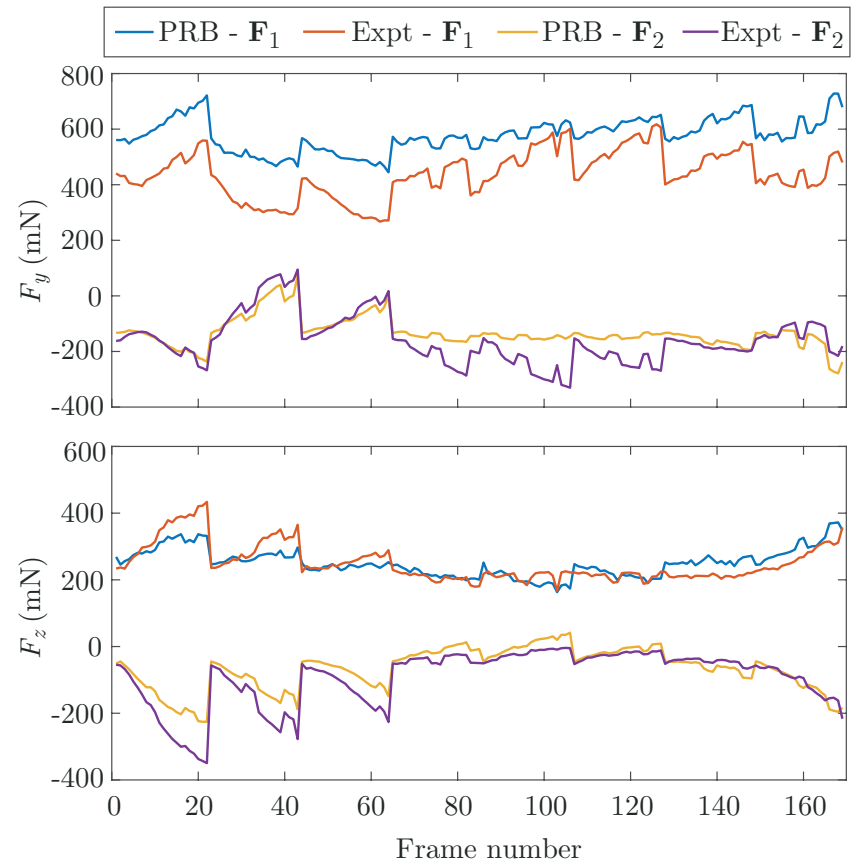

Figure 7: Comparison of constraint forces obtained from experiments (Expt) to those calculated using the pseudo rigid body model (PRB). Displacement constraints are applied at two points along the length of the manipulator, and the corresponding reaction forces are named $\mathbf{F}_{1}$ and $\mathbf{F}_{2}$. The top plot shows the forces in the direction of the $y$-axis (horizontal plane), and the bottom one shows the force in the direction of the $z$-axis (vertical plane).

parameter optimization. The errors could also be due to deformation of the fixtures during the experiment, which means the displacement constraints are not maintained perfectly. For the manipulator shape, it was noticed that a major portion of the error was in the first PRB segment (between $\mathbf{P}_{0}$ and $\mathbf{P}_{1}$ ) due to the manipulator not being fully constrained at the base. It is also noticeable in Fig. 9 that the red dots obtained from the stereo-vision tracking system do not follow the shape of the manipulator perfectly, especially close to the fixed base. In spite of these issues, the estimated reaction forces and deformed shapes are close to the experimental results. The estimation of contact forces described here requires knowledge of the positions of the constraints $\left(\mathbf{P}_{1}\right.$ and $\left.\mathbf{P}_{2}\right)$, which may not be readily available during application. If the manipulator is enclosed in a conduit, this can be addressed by providing the shape of the conduit and restricting the shape of the manipulator to stay within the permitted volume.

It is possible to increase the number of segments to achieve higher accuracy, but that will require a trade-off with computation time (In the work shown here, the single PRB model takes $6.8 \mathrm{~ms}$ while the combined model with external forces requires $174.5 \mathrm{~ms}$ on average for one calculation on a $8 \mathrm{~GB}, 3.60 \mathrm{GHz}$ Intel i7 machine running Windows 10). Nonlinear spring stiffnesses can be introduced if the accuracy using linear springs is considered to be insufficient. A larger pool 


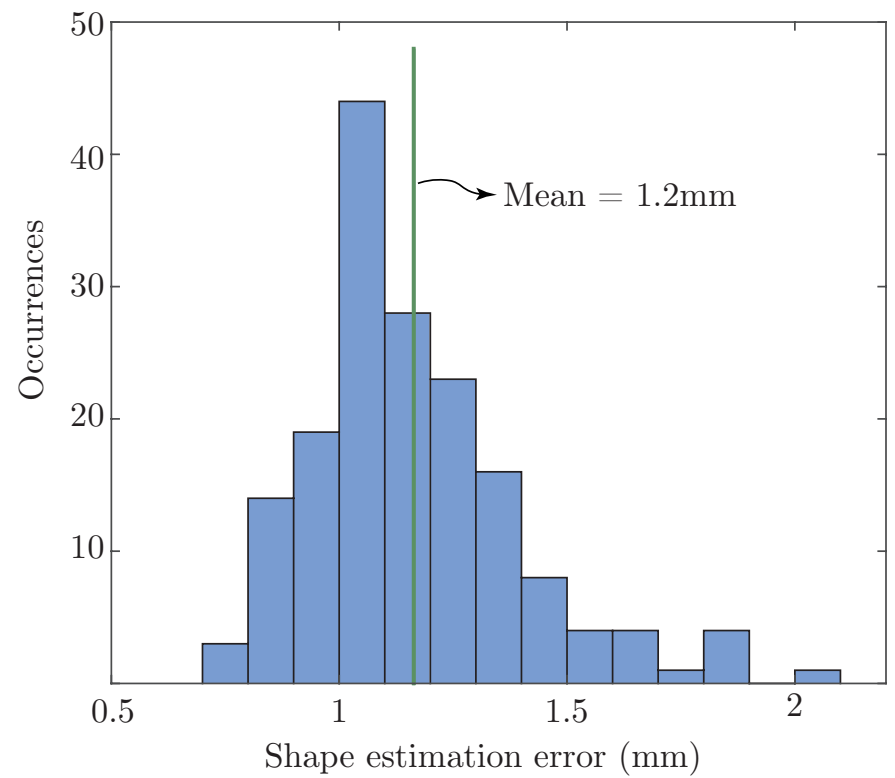

Figure 8: A histogram of the error in shape estimation of the manipulator by the PRB model under multiple loads. The chart denotes the average distance between a spline through the PRB points and the tracking data obtained from experiments, across 169 occurrences.

of data for optimization will also help in reducing error. The model can also incorporate more complex behavior - for instance, a manipulator with an initial curvature can be defined with non-zero equilibrium angles. Twisting, shear and extension effects can also be integrated using suitable mechanical elements. Gravity has been included as a point load at the tip, but a distributed load profile can also be introduced with a bias in the vertical plane of the model.

\section{CONCLUSIONS AND FUTURE WORK}

In this study, a framework is presented for the analysis of continuum manipulators using PRB models under quasi-static conditions. The method is applied for estimating the reaction forces and shape of a manipulator subjected to environmental constraints. An optimized PRB model is developed for a single segment, and the same parameter values are used for analyzing a manipulator with multiple loads exerted on it. The model shows good agreement with experiments in estimating the forces $(61 \mathrm{mN}$ mean error) and predicting the deformed shape of the manipulator (1.2 $\mathrm{mm}$ error in shape along length), while allowing for fast computation. While the PRB model is not derived from fundamental principles of elastic rods, the adaptability of the optimization approach allows the same framework to be used for various types of continuum manipulators.

In the future, this approach will be applied to model the interaction of a manipulator with its environment, and extended to closed-loop control of manipulator motion. The use of the model for transient analysis and timevarying loads will also be studied. The model can also be combined with other 

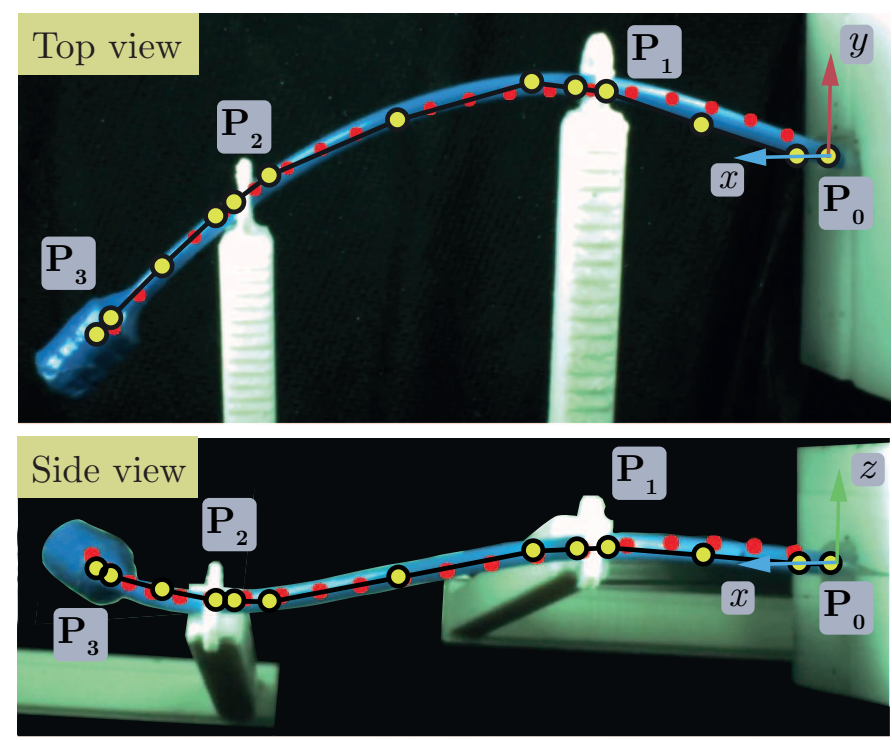

(a) Frame 40
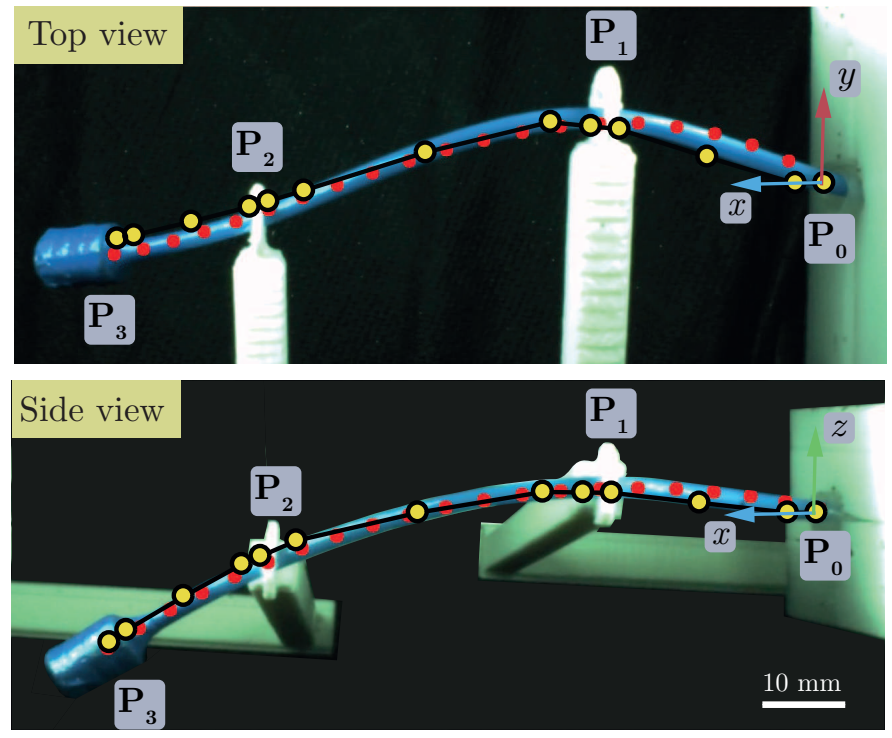

(b) Frame 97

Figure 9: The shape of the manipulator calculated using the pseudo rigid body model (black lines with yellow circles) is overlaid with the photographs from experiment. The manipulator (in blue) is fixed at $\mathbf{P}_{0}$ and displacement constraints are applied at $\mathbf{P}_{1}$ and $\mathbf{P}_{2}$ using the supports shown in white. The forces at these points are measured using two 3-axis force sensors (not shown). The manipulator is divided into three segments, and each segment is approximated by a PRB model with four rigid links. The red dots are used to define a polynomial curve for the manipulator shape, obtained using a tracking method similar to Ref. [29]. The magnetic tip is at $\mathbf{P}_{3}$ and is actuated by an external magnetic field using an array of electromagnetic coils. The results of two instances of the experiment are shown, corresponding to frames 40 and 97 in Fig. 7. 
sensing modalities, such as Fiber Bragg Grating Sensors (FBGS) or ultrasound scanning to be used for surgical applications.

\section{Acknowledgements}

This research has received funding from the European Research Council (ERC) under the European Unions Horizon 2020 Research and Innovation programme (Grant Agreement \#638428 - project ROBOTAR).

\section{References}

[1] A. A. Transeth, K. Y. Pettersen, P. Liljebck, A survey on snake robot modeling and locomotion, Robotica 27 (7) (2009) 999-1015. doi:10.1017/S0263574709005414.

[2] L. Margheri, C. Laschi, B. Mazzolai, Soft robotic arm inspired by the octopus: I. From biological functions to artificial requirements, Bioinspiration \& Biomimetics 7 (2) (2012) 025004. doi:10.1088/1748-3182/7/2/025004.

[3] J. Burgner-Kahrs, D. C. Rucker, H. Choset, Continuum Robots for Medical Applications: A Survey, IEEE Transactions on Robotics 31 (6) (2015) 1261-1280. doi:10.1109/TRO.2015.2489500.

[4] C. Majidi, Soft Robotics: A Perspective - Current Trends and Prospects for the Future, Soft Robotics 1 (1) (2013) 5-11. doi:10.1089/soro.2013.0001.

[5] R. J. Webster, B. A. Jones, Design and Kinematic Modeling of Constant Curvature Continuum Robots: A Review, Design and Kinematic Modeling of Constant Curvature Continuum Robots: A Review, The International Journal of Robotics Research 29 (13) (2010) 1661-1683. doi: $10.1177 / 0278364910368147$.

[6] K. Xu, N. Simaan, Analytic Formulation for Kinematics, Statics, and Shape Restoration of Multibackbone Continuum Robots Via Elliptic Integrals, Journal of Mechanisms and Robotics 2 (1) (2010) 011006. doi:10.1115/1.4000519.

[7] D. Trivedi, A. Lotfi, C. D. Rahn, Geometrically Exact Models for Soft Robotic Manipulators, IEEE Transactions on Robotics 24 (4) (2008) 773780. doi:10.1109/TRO.2008.924923.

[8] D. C. Rucker, B. A. Jones, R. J. Webster, A Geometrically Exact Model for Externally Loaded Concentric-Tube Continuum Robots, IEEE Transactions on Robotics 26 (5) (2010) 769-780. doi:10.1109/TRO.2010.2062570.

[9] W. Tang, T. R. Wan, D. A. Gould, T. How, N. W. John, A Stable and RealTime Nonlinear Elastic Approach to Simulating Guidewire and Catheter Insertions Based on Cosserat Rod, IEEE Transactions on Biomedical Engineering 59 (8) (2012) 2211-2218. doi:10.1109/TBME.2012.2199319. 
[10] J. Till, C. E. Bryson, S. Chung, A. L. Orekhov, D. C. Rucker, Efficient computation of multiple coupled Cosserat rod models for real-time simulation and control of parallel continuum manipulators, in: 2015 IEEE International Conference on Robotics and Automation (ICRA), 2015, pp. 5067-5074. doi:10.1109/ICRA.2015.7139904.

[11] H. Mochiyama, Model validation of discretized spatial closed elastica, in: 2016 IEEE/RSJ International Conference on Intelligent Robots and Systems (IROS), 2016, pp. 5216-5223. doi:10.1109/IROS.2016.7759767.

[12] J. Back, T. Manwell, R. Karim, K. Rhode, K. Althoefer, H. Liu, Catheter contact force estimation from shape detection using a realtime Cosserat rod model, in: 2015 IEEE/RSJ International Conference on Intelligent Robots and Systems (IROS), 2015, pp. 2037-2042. doi:10.1109/IROS.2015.7353647.

[13] E. Coevoet, A. Escande, C. Duriez, Optimization-Based Inverse Model of Soft Robots With Contact Handling, IEEE Robotics and Automation Letters 2 (3) (2017) 1413-1419. doi:10.1109/LRA.2017.2669367.

[14] Z. Zhang, J. Dequidt, J. Back, H. Liu, C. Duriez, Motion Control of Cable-Driven Continuum Catheter Robot Through Contacts, IEEE Robotics and Automation Letters 4 (2) (2019) 1852-1859. doi:10.1109/LRA.2019.2898047.

[15] L. L. Howell, Compliant Mechanisms, John Wiley \& Sons, New York, NY, 2001.

[16] V. K. Venkiteswaran, O. A. Turkkan, H.-J. Su, Speeding Up Topology Optimization of Compliant Mechanisms With a Pseudorigid-Body Model, Journal of Mechanisms and Robotics 9 (4) (2017) 041007. doi:10.1115/1.4035992.

[17] M. Khoshnam, R. V. Patel, A pseudo-rigid-body 3r model for a steerable ablation catheter, in: 2013 IEEE International Conference on Robotics and Automation, 2013, pp. 4427-4432. doi:10.1109/ICRA.2013.6631205.

[18] T. Greigarn, M. C. Çavuşoğlu, Pseudo-rigid-body model and kinematic analysis of MRI-actuated catheters, in: 2015 IEEE International Conference on Robotics and Automation (ICRA), 2015, pp. 2236-2243. doi:10.1109/ICRA.2015.7139495.

[19] T. Greigarn, R. Jackson, T. Liu, M. C. Çavuşoğlu, Experimental validation of the pseudo-rigid-body model of the MRI-actuated catheter, in: 2017 IEEE International Conference on Robotics and Automation (ICRA), 2017, pp. 3600-3605. doi:10.1109/ICRA.2017.7989414.

[20] R. J. Roesthuis, S. Misra, Steering of Multisegment Continuum Manipulators Using Rigid-Link Modeling and FBG-Based Shape 
Sensing, IEEE Transactions on Robotics 32 (2) (2016) 372-382. doi:10.1109/TRO.2016.2527047.

[21] S. Huang, D. Meng, Y. She, X. Wang, B. Liang, B. Yuan, Statics of Continuum Space Manipulators With Nonconstant Curvature via Pseudorigid-Body 3r Model, IEEE Access 6 (2018) 70854-70865. doi:10.1109/ACCESS.2018.2881261.

[22] J. Edelmann, A. J. Petruska, B. J. Nelson, Magnetic control of continuum devices, The International Journal of Robotics Research 36 (1) (2017) 6885 .

[23] C. Heunis, J. Sikorski, S. Misra, Improved magnetic steering, actuation, and image-guided surgical instruments, IEEE Robotics \& Automation Magazine 1070 (9932/18) (2018) 2.

[24] G. Tholey, J. P. Desai, A. E. Castellanos, Force Feedback Plays a Significant Role in Minimally Invasive Surgery, Annals of Surgery 241 (1) (2005) 102109. doi:10.1097/01.sla.0000149301.60553.1e.

[25] S. Ernst, Initial Experience With Remote Catheter Ablation Using a Novel Magnetic Navigation System: Magnetic Remote Catheter Ablation, Circulation 109 (12) (2004) 1472-1475. doi:10.1161/01.CIR.0000125126.83579.1B.

[26] J. Sikorski, I. Dawson, A. Denasi, E. E. G. Hekman, S. Misra, Introducing BigMag - A novel system for 3d magnetic actuation of flexible surgical manipulators, in: 2017 IEEE International Conference on Robotics and $\mathrm{Au}-$ tomation (ICRA), 2017, pp. 3594-3599. doi:10.1109/ICRA.2017.7989413.

[27] R. M. Murray, Z. Li, S. S. Sastry, S. S. Sastry, A Mathematical Introduction to Robotic Manipulation, CRC Press, 1994.

[28] V. K. Venkiteswaran, H.-J. Su, A parameter optimization framework for determining the pseudo-rigid-body model of cantilever-beams, Precision Engineering 40 (2015) 46-54. doi:10.1016/j.precisioneng.2014.10.002.

[29] D. B. Camarillo, K. E. Loewke, C. R. Carlson, J. K. Salisbury, Vision based 3-d shape sensing of flexible manipulators, in: Robotics and Automation, 2008. ICRA 2008. IEEE International Conference on, IEEE, 2008, pp. 2940-2947. 